\title{
Digital Fabric Libraries: Managing Digital Assets
}

\author{
Uikyung Jung, and Cynthia Istook, North Carolina State University
}

\section{Introduction}

Industry executives predict that digitization will be the next sourcing concern in this fastchanging and uncertain environment (Berg et al., 2017). The digital transformation of the fashion and apparel industry enables transparency, predictability, and efficiency in the global supply chain (Berg et al., 2017; Mageean, 2019). Some pioneering brands have made progress with partial or company-wide workflow digitization and exploration of 3D virtual garment technology in long-term strategic planning in the recent decade. The one key element required is a realistic $3 \mathrm{D}$ representation and digitized material that make $3 \mathrm{D}$ garments useful as decision-making tools (Le, 2017).

As product design and development workflow are becoming digitized, advanced digital visualization technologies for material came into the world, such as fabric scanner, 3D rendering, fabric property test kit, and virtual light booth. They can empower more efficient decisions regarding a product's silhouette, material, design details, and colorways throughout the overall supply chain (Prahl, 2017). This paper brought information together on currently available digital material library platforms and fabric scanners from academic journals, company websites, and industry magazines to make a cornerstone of managing digital material for the 3D virtual product. Moreover, the authors suggested potential spaces for improvements to take an essential step toward enhancing the reliability and accuracy of the 3D virtual garment simulation and gaining full acceptance of the introduced digital material visualization technologies for the software.

\section{Issues of Preexisting Fabric Library}

In the fashion and apparel industry, fabric management is further challenged by a multitude of material sources from mills, agents, or converters all over the world. A brand has to work with hundreds of new fabrics each season, and the fabrics are shipped back and forth between the brand and suppliers to communicate (Mageean, 2018). Each brand often has its own storage facilities for fabric swatches, which are referred to as "fabric libraries." The fabric library acts as a key information source and is reviewed by designers, buyers, merchandisers, and material teams in the firm to develop new collections and make merchandising and sourcing plans. The fabric information must be input manually and updated frequently to register new fabric swatches, and fabric swatches should be placed and organized in accordance with a classification system. A study conducted by Moon and Ngai (2010) indicates that a desirable system for a fabric library should possess functionalities of item searching, item filtering, a 
suggestion of alternative items, integrating visual and technical data in a single presentation, and share information in real-time for better efficiency and work performance.

\section{Online Database Platforms for Digital Materials}

The demand for a digital material library has emerged to connect brands, suppliers, and manufacturers in the world and provide greater consistency and applicability of digitized material into different design software. Moreover, the desire to facilitate a reduction in the generation of physical material swatches and garments prototypes led to the establishment of digital material library platforms such as Material Exchange and Swatchbook in 2018. Material Exchange and Swatchbook are online database platforms that enable designers, product developers, and material suppliers to collaborate, visualize and browse the raw materials for collections within a secure environment (Material Exchange, n.d.; Prahl, 2017; Swatchbook, n.d.).

The benefits that are provided by digital fabric library platforms are multifold. First, the platform enables users to browse digital materials with integrated information about their physical properties, country of origin, lead times, supplier, and pricing information that suppliers and brands can provide. Second, brands and suppliers can share a cloud environment and collaborate about planning material collection. Third, brands can create folders and archive seasonal collections and their swatch data. Fourth, the platforms provide digitized fabric texture images and allow them to apply to a 3D model with scale and color adjustment before and after getting physical samples. Finally, users can export a report of material specs and pictures into PDM, PLM, and 3D virtual garment software (Material Exchange, n.d.; Prahl, 2017; Swatchbook, n.d.).

\section{Capturing Material Digitally with Fabric Scanners}

In the 3D virtual garment software, fabric texture images could be imported and embedded into 3D products. Material visual quality and drape representation of 3D garments are essential for visual inspection and design detail review. For example, users may want to zoom in on a simulated garment to observe overall fit, drapability, textile design, and fabric construction. High-resolution images can be acquired with a flatbed scanner; however, image editing software must be utilized to recreate further the visual property maps with appropriate size, resolution, and repeat.

To address this, sophisticated fabric scanners and interconnected software for capturing photorealistic texture images with high DPI have been released. For a heightened realism of a material image capture, Vizoo and X-Rite invented scanning cameras and a lightbox for scanning. Their high-quality scanners can obtain visual properties by considering several texture maps of color, structure, reflectivity, transparency, etc., and it helps to enhance the accuracy and quality of recreated virtual materials under different lighting conditions (Mageean, 2017; Steinhausen et al., 2018). Moreover, the interconnected software enables users to generate a seamless repeat and 
edit individual texture maps. The scanned visual material data and images can be exported and imported in 3D virtual garment software, 3D rendering software, and digital material database platforms.

\section{Proposal}

The current digital material library platforms tend to focus on the visual aspect of material and embedding material images into 3D product shape before and after getting physical samples. Both Material Exchange and Swatchbook provide fabric images that are draped over a ball, and the 3D view is operable by spinning around and zooming in to see the texture. However, in the fabric images, the draped shape is the same throughout all samples, and only texture appearance alters, which isn't real-to-life. The current platforms only provide fabric information on material content, width, and thickness. As long as the platforms don't reflect fabric properties in the draped image of the fabric and 3D model applications, the most appropriate product decisions cannot be made using these technologies. Including and combining more fabric properties could make the 3D drape image more accurate in a physical garment, leading to optimal decision making. In the long term, as the digital fabric library grows, collected fabric properties data could be utilized to predict the drape of new fabrics without the physical process by applying artificial intelligence.

Furthermore, the file type of virtual fabrics acquired from fabric scanners could be standardized so that relevant data would be available regardless of the 3D CAD system. This is a significant barrier to adopting technologies that could help speed product to market in more sustainable ways. Finally, one standardized method of obtaining and communicating fabric properties and drape images could be established for coherent visualization throughout the digital supply chain.

Page 3 of 4

(c) 2020 The author(s). Published under a Creative Commons Attribution License (https://creativecommons.org/licenses/by/4.0/), which permits unrestricted use, distribution, and reproduction in any medium, provided the original work is properly cited.

ITAA Proceedings, \#77 - https://itaaonline.org 


\section{References}

Berg, A., Hedrich, S., Lange, T., Magnus, K. H., \& Mathews, B. (2017). The Apparel Sourcing Caravan's Next Stop: Digitization. McKinsey Apparel CPO Survey 2017. McKinsey Apparel, Fashion \& Luxury Group

Le, K. (2017). Virtual Textiles: Making Realistic Fabrics in 3D. AATCC Review Vol. 17, No. 3 May/June 2017

Mageean, L. (2017). Vizoo xTex Advertorial; Bringing your digital fabrics to life. Retrieved from https://www.whichplm.com/vizoo-xtex-advertorial-bringing-digital-fabrics-life/

Mageean L., (2018). The designer's struggle with materials. whichPLM. Retrieved from https://www.whichplm.com/the-designers-struggle-with-materials/

Material Exchange. (n.d.). Retrieved from https://material-exchange.com/

Moon, K. L., \& Ngai, E. W. (2010). R\&D framework for an intelligent fabric sample management system. International Journal of Operations \& Production Management.

Prahl, A. (2017 December). 3D digital technologies: Material focus. Innovation In Textiles. Retrieved from https:/www.innovationintextiles.com/3d-digital-technologies-material-focus/

Swatchbook. (n.d.). Retrieved from https://www.swatchbook.us/\#home

Steinhausen, H. C., den Brok, D., Merzbach, S., Weinmann, M., \& Klein, R. (2018). Data-driven Enhancement of SVBRDF Reflectance Data. In VISIGRAPP (1: GRAPP) (pp. 273-280). 